\title{
Atypical Hemorrhagic Brain Metastases Mimicking Cerebral Microbleeds
}

\author{
Sulki Lee, MD, Heewon Bae, MD, Unkyu Yun, MD, Inha Hwang, MD, Seung Min Kim, MD, PhD \\ Department of Neurology, Veterans Health Service Medical Center, Seoul, Korea
}

A 65-year-old man with hypertension and diabetes presented with a history of speech disturbance and gait disturbance of several months duration. He had a 50 pack-year smoking history. Neurologic examination revealed dysarthria and truncal ataxia. Initial vital signs and routine laboratory tests were unremarkable. Although the axial fluid attenuated inversion recovery, $\mathrm{T} 1$ and $\mathrm{T} 2$-weighted brain magnetic resonance imaging (MRI) revealed no definite evidence of abnormality (Fig. 1A), the T2-weighted gradient echo (GRE) images revealed multiple, hypo-intense lesions in the cortical and subcortical areas (Fig. 1B). In view of the particular patient's multiple risk factors for stroke, the presence of asymptomatic cerebral microbleeds were highly suspected. There were, however no findings to suggest microbleeds on a brain MRI, conducted 8 months earlier to evaluate a postural hand tremor (Fig. 1C).

Therefore, newly developed multiple microbleeds raised a suspicion of hemorrhagic brain metastasis, leading to a focus on finding, identifying and evaluating a potentially hidden malignancy. An additional, contrast-enhanced brain MRI revealed multiple, enhanced, nodular lesions (Fig. 1D). A chest computed tomography revealed a tumor in the lower lobe of the right lung with pleural effusion and multiple metastatic lymph nodes (Fig. 1E). A bronchoscopic lung biopsy was conducted and subsequently, poorly-differentiated adenocarcinoma was diagnosed. Two months after the initial diagnosis, the patient returned with report of a severe headache and nausea. A follow-up MRI revealed prominent multifocal enhancing nodules suggestive of progression of metastasis (Fig. 1F).

Metastases that are characterized by hemorrhage as (one) classic clinical sign include malignancies such as melanoma, choriocarcinoma, renal cell carcinoma, thyroid, lung and breast cancer. Of all hemorrhagic metastases, however, lung and breast cancers are the most common etiologies due to their higher overall prevalence. ${ }^{1}$ Previous studies have demonstrated that hemorrhagic metastasis contain

\author{
Received September 19, 2017 \\ Revised October 12, 2017 \\ Accepted October 16, 2017 \\ Corresponding Author: \\ Seung Min Kim, MD, PhD \\ Department of Neurology, Veterans \\ Health Service Medical Center, 53 \\ Jinhwangdo-ro 61-gil, Gangdong-gu, \\ Seoul 05368, Korea \\ Tel: +82-2-2225-1166 \\ Fax: +82-2-2225-1327 \\ E-mail: kh21762@hanmail.net
}

Copyright $\odot 2017$ The Korean Neurocritical Care Society

cc This is an Open Access article distributed under the terms of the Creative Commons Attribution Non-Commercial License (http://creativecommons.org/licenses/by$\mathrm{nc} / 4.0$ ) which permits unrestricted non-commercial use, distribution, and reproduction in any medium, provided the original work is properly cited. 

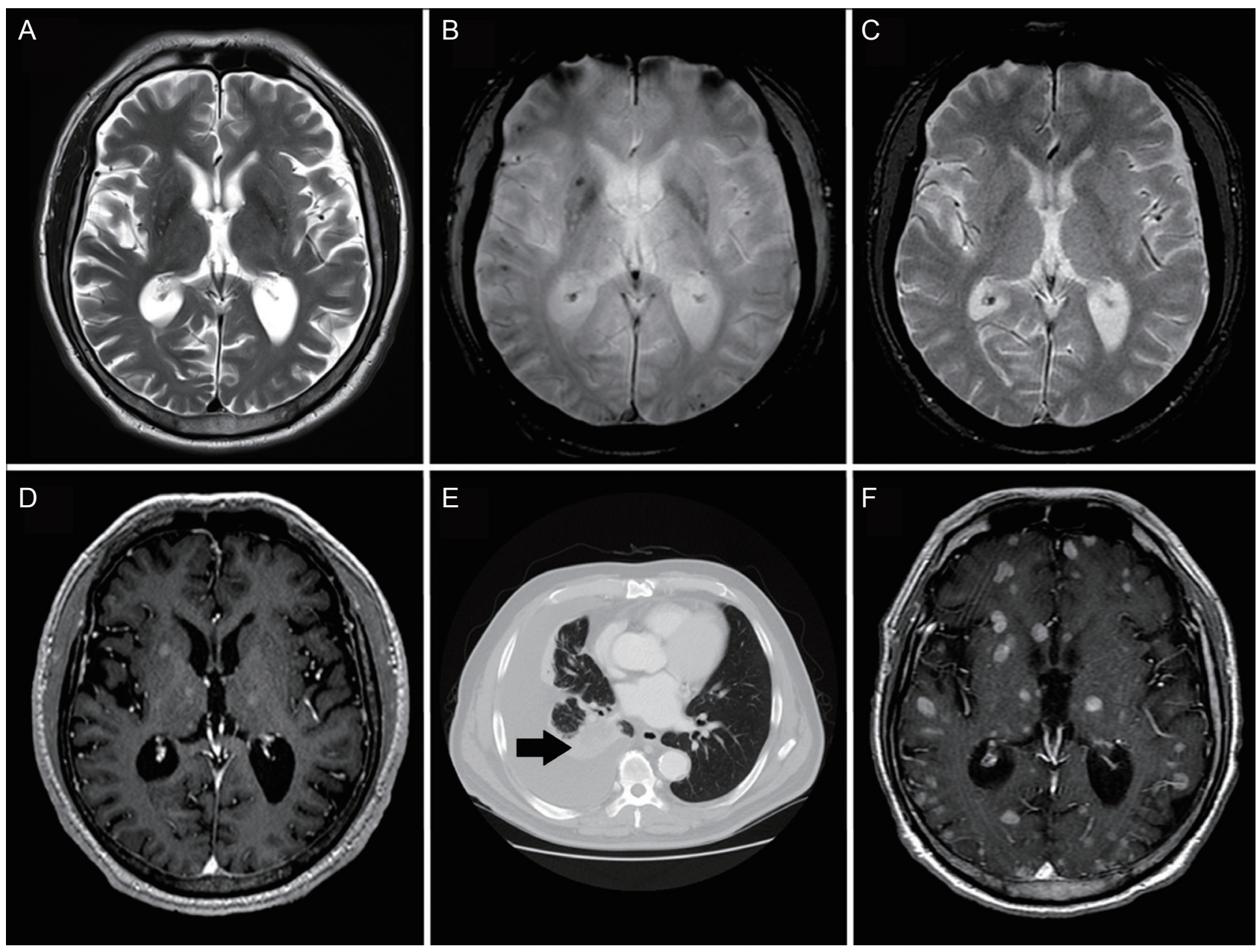

Figure 1. The Axial T2-weighted magnetic resonance imaging (MRI) reveals no definitive abnormal findings (A). Axial T2-weighted gradient echo (GRE) image demonstrates multiple hypointense, hemorrhagic lesions in the cortical and subcortical areas (B). Axial T2weighted GRE image obtained 8 months before symptom onset reveals no evidence of microbleeds (C). Additional contrast-enhanced T1-weighted MRI reveals multiple, enhanced, nodular lesions (D). Chest computed tomography demonstrates lung mass (arrow) in the right lower lobe with pleural effusion (E). Follow up contrast-enhance T1-weighted MRI demonstrates prominent multifocal enhancing nodules suggestive of progression of metastasis (F).

significantly higher levels of vascular endothelial growth factor (VEGF) and matrix metalloproteinases (MMP), when these malignancies were compared with non-hemorrhagic tumors. ${ }^{2,3}$ The over-expressed VEGF and MMP increase the permeability of the blood vessels, resulting in microhemorrhages. In case of metastatic melanoma (that can appear hypointense on GRE), the specific malignancy can often be distinguished from cerebral microbleed by the concomitant presence of $\mathrm{T} 1$ hyperintensity (caused by the melanin). However, it is difficult to differentiate between cerebral microbleed and hemorrhagic metastasis due to other primary cancers.

If there is no history of cancer or contrast-enhanced MRI, it can be challenging to differentiate hemorrhagic metastasis from cerebral microbleeds. ${ }^{4}$ Detection by contrast enhanced methods may depend on the ratio of tumor vascularity to size. However, detection by T2-weighted gradientecho MRI depends on magnetic susceptibility. Thus, T2weignted GRE MRI can detect much smaller hemorrhagic metastases than the smallest tumor that can be detected on contrast-enhanced MRI. ${ }^{5,6}$ In addition, because there is a relatively restricted number of kinds of primary cancers 
that cause hemorrhagic metastases, GRE can enable identification of the primary cancer. Therefore, it is necessary to conduct brain MRI scans to include various sequences including GRE in to diagnose small hemorrhagic metastasis that may be undetected on T1-enhanced images.

\section{REFERENCES}

1. Fink KR, Fink JR. Imaging of brain metastases. Surg Neurol Int 2013;4(Suppl 4):S209-19.

2. Jung S, Moon KS, Jung TY, Kim IY, Lee YH, Rhu HH, et al. Possible pathophysiological role of vascular endothelial growth factor (VEGF) and matrix metalloproteinases (MMPs) in metastatic brain tumor-associated intracerebral hemorrhage. / Neurooncol 2006;76:257-63.

3. Navi BB, Reichman JS, Berlin D, Reiner AS, Panageas KS, Segal $A Z$, et al. Intracerebral and subarachnoid hemorrhage in patients with cancer. Neurology 2010;74:494-501.

4. Tsushima Y, Aoki J, Endo K. Brain microhemorrhages detected on T2*-weighted gradient-echo MR images. AJNR Am J Neuroradiol 2003;24:88-96.

5. Blitstein MK, Tung GA. MRI of cerebral microhemorrhages. AJR Am / Roentgenol 2007;189:720-5

6. Murata Y, Itoh S, Morio K, Sasaki T, Mizobuchi H, Shimizu K, et al. Hemorrhagic cerebral metastases from thyroid cancer on T2*-weighted gradient echo MRI. Mag Reson Imaging 2004;22:435-9. 